
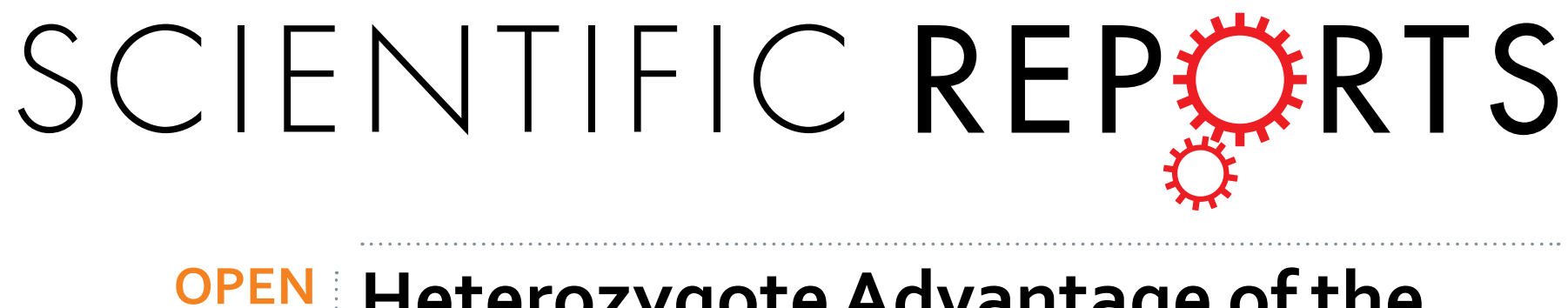

\title{
Heterozygote Advantage of the rs3794624 Polymorphism in CYBA for Resistance to Tuberculosis in Two Chinese Populations
}

Received: 08 June 2016 Accepted: 07 November 2016 Published: 30 November 2016
Qianqian Liv ${ }^{1,2,3, *}$, Shouquan Wu ${ }^{1, *}$, Miao Xue ${ }^{1, *}$, Andrew J. Sandford ${ }^{4}$, Jingcan Wu ${ }^{1}$, Yu Wang ${ }^{1}$, Guo Chen ${ }^{5}$, Chuanmin Tao ${ }^{3}$, Yin Tang ${ }^{6}$, Yulin Feng ${ }^{1}$, Jun Luo ${ }^{7}$ \& Jian-Oing He ${ }^{1}$

Phagocyte Nicotinamide Adenine Dinucleotide Phosphate (NADPH) oxidase complex is a key enzyme that catalyzes the production of reactive oxygen species, which mediate oxygen-dependent killing of microorganisms, such as Mycobacterium tuberculosis. P22phox, encoded by CYBA, is the key regulatory subunit of NADPH oxidase. Our study aimed to investigate the association of CYBA polymorphisms with susceptibility to tuberculosis. Three SNPs (rs9932581, rs3794624 and rs4673) were genotyped in the discovery cohort composed of Chinese Han individuals. We found that the A allele of rs3794624 was a significant protective factor against tuberculosis (GA vs. GG: OR $=0.74,95 \% \mathrm{Cl} 0.57-0.96$; GA vs. $\mathrm{GG}+\mathrm{AA}: \mathrm{OR}=0.73,95 \% \mathrm{Cl} 0.56-0.95)$, which was then replicated in the Chinese Tibetan population (GA vs. GG: $\mathrm{OR}=0.68,95 \% \mathrm{Cl} 0.51-0.92 ; \mathrm{AA}+\mathrm{GA}$ vs. GG: $\mathrm{OR}=0.70,95 \% \mathrm{Cl} 0.52-0.93$; GA vs. GG+AA: $\mathrm{OR}=0.68,95 \% \mathrm{Cl} 0.51-0.92$ ). Meta-analysis including both cohorts identified overdominance as the best genetic model and provided robust evidence for the protective effect of the rs $3794624 \mathrm{GA}$ genotype against tuberculosis without any evidence of heterogeneity (GA vs. GG+AA: $O R=0.71,95 \%$ $\mathrm{Cl}$ 0.58-0.86). Our study found an association between the GA genotype of rs 3794624 in CYBA with decreased tuberculosis susceptibility in two Chinese populations. Further analyses are needed to reveal the potential function of this SNP.

Tuberculosis (TB), caused by Mycobacterium tuberculosis (MTB), claims 1.5 million lives annually and remains the most important public health problem in developing countries, especially China ${ }^{1}$. According to epidemiological data, only a small fraction (5-15\%) of the population infected with MTB will develop clinically active disease during their lifetime ${ }^{2}$. Susceptibility to TB is likely due to variation in MTB virulence, host genetic factors as well as environmental determinants ${ }^{3-5}$. As acquired immunodeficiency syndrome and other immune-compromising conditions significantly increase the risk of developing $\mathrm{TB}$, it is clear that protective immune responses function efficiently to combat the invading MTB in the majority of individuals ${ }^{6}$. In recent years, a large number of genetic association studies have been conducted that have mainly focused on host immunity against MTB, and the results of these studies have enhanced our understanding of the pathogenesis of $\mathrm{TB}^{7}$.

The innate immune system serves as the first line of defense against MTB infection that recognizes and phagocytizes invading pathogen, rapidly killing the engulfed microbe, limits its growth and proliferation, induces apoptosis, and promotes the secretion of chemokines and pro-inflammatory cytokines ${ }^{6}$. It is worth noting that reactive oxygen species (ROS) produced by the phagocyte Nicotinamide Adenine Dinucleotide Phosphate (NADPH) oxidase complex during the respiratory burst is suggested to play a critical role throughout the entire innate immune

${ }^{1}$ Department of Respiratory and Critical Care Medicine, West China Hospital, Sichuan University, Chengdu, Sichuan, China. ${ }^{2}$ Department of Respiratory Diseases, Chengdu Municipal First People's Hospital, Chengdu, Sichuan, China. ${ }^{3}$ Department of Laboratory Medicine, West China Hospital, Sichuan University, Chengdu, Sichuan, China. ${ }^{4}$ Centre for Heart Lung Innovation, University of British Columbia and St. Paul's Hospital, Vancouver, BC, Canada. ${ }^{5}$ Division of Geriatrics, Sichuan Provincial People's Hospital, Chengdu, Sichuan, China. ${ }^{6}$ State Key Laboratory of Oral Disease, West China School \& Hospital of Stomotology, Sichuan University, Chengdu, Sichuan, China. ${ }^{7}$ Division of Infectious Diseases, People's Hospital of Aba Tibetan Autonomous Prefecture, Maer, Sichuan, China. *These authors contributed equally to this work. Correspondence and requests for materials should be addressed to J.L. (email: 1643542114@qq.com) or J.-O.H. (email: jianqhe@gmail.com) 
defenses, contributing to the clearance of $\mathrm{MTB}^{8}$. Dysfunction of the phagocyte NADPH oxidase complex leads to a primary immunodeficiency, chronic granulomatous disease, which exhibits an especially high risk of clinical TB and BCG complications ${ }^{9-13}$. Thus, there is a strong rationale for the investigation of phagocyte NADPH oxidase complex function with respect to TB susceptibility.

Phagocyte NADPH oxidase complex is comprised of six subunits, including two transmembranous subunits (gp91phox and p22phox), three cytosolic subunits (p67phox, p47phox, and p40phox), and a GTP-binding protein (Rac1 or 2$)^{14}$. Of these subunits, p22phox (encoded by CYBA) is the key regulatory subunit that acts as the final transporter in the electron-transfer chain from NADPH to molecular oxygen ${ }^{15}$. However, to our knowledge no studies have evaluated the association of polymorphisms in CYBA with TB susceptibility, although polymorphisms in this gene have been reported to exert an influence on several ROS-associated diseases, such as hypertension, coronary heart disease and chronic obstructive pulmonary disease $\mathrm{e}^{16-18}$. Therefore, the aim of our study was to determine the role of polymorphisms in CYBA in susceptibility to TB.

\section{Materials and Methods}

Study population. In the discovery cohort, a total of 1244 unrelated ethnic Han Chinese (636 TB patients and 608 healthy controls) were recruited between July 2012 and August 2014 from the West China Hospital of Sichuan University. An independent replication cohort was composed of Chinese Tibetans (613 TB patients and 603 healthy controls) consecutively recruited between February 2013 and August 2015 from the People's Hospital of the Aba Tibetan Autonomous Prefecture. The study protocol was approved by the ethics committee of West China Hospital and People's Hospital of Aba Tibetan Autonomous Prefecture. Methods were carried out in accordance with the approved guidelines. Written informed consent was obtained from each subject. Demographic characteristics of all participants were collected from a detailed questionnaire.

The diagnosis of TB was based on the following criteria: culture positive for MTB and/or smear positive for MTB and/or histopathological findings of TB disease and/or clinical and radiographic presentation consistent with $\mathrm{TB}$, with positive response to anti-TB therapy. The healthy control groups were selected from individuals who presented to the outpatient department of the West China Hospital or the People's Hospital of the Aba Tibetan Autonomous Prefecture for annual physical examination, without active TB, without history of TB and matched with cases by gender and age.

Individuals with the following conditions were excluded from the study: human immunodeficiency virus infection, autoimmune disease, cancer, primary immunodeficiency, treatment with immunosuppressive drugs, diabetes mellitus.

SNP selection. SNPs in the region between 3,000 base pairs upstream and 2000 base pairs downstream of CYBA were screened based on literature review ${ }^{16,19,20}$ and in silico functional prediction from the FuncPred (http:// snpinfo.niehs.nih.gov/snpinfo/snpfunc.htm) and Regulome DB (http://regulome.stanford.edu/) databases. Only those SNPs with significant disease associations and potential effects on function were included in our study. As a result, three SNPs (rs9932581 T > C, rs3794624 G > A and rs4673 T > C) were selected. rs9932581 is located in the 5' promoter region of $C Y B A$, at position -930 from the start codon, and was reported to be associated with hypertension and coronary artery disease ${ }^{16,21}$. The rs 9932581 polymorphism was demonstrated by mutagenesis experiments to result in altered promoter activity. rs3794624, located in intron 1, was found to be a strong contributing factor to the Ankle-Brachial Index $\left(p=6.3 \times 10^{-5}\right)$ and have moderate effects on postmenopausal breast cancer risk ${ }^{19,22}$. And both above-mentioned SNPs were predicted to be potential transcription factor binding sites by the FuncPred and Regulome DB databases (rs9932581 T > C scored 4; rs3794624 G > A scored 2a). rs4673 was reported to be associated with essential arterial hypertension, coronary artery disease, and type 2 diabetes mellitus and so on ${ }^{20,23,24}$. It is a non-synonymous SNP located in exon 4, that leads to a histidine/tyrosine substitution and was shown to have an influence on both basal and NADH-stimulated superoxide production ${ }^{20}$. The FuncPred database also reported that the substitution was possibly damaging by Polyphen analysis.

Genotyping. We collected venous blood samples $(4-5 \mathrm{ml})$ in EDTA tubes (BD Vacutainers, Franklin Lakes, NJ, USA) from each subject. Genomic DNA was isolated using the AxyPrep DNA Blood kit (Axygen Scientific Inc, Union City, CA, USA), according to the manufacturer's instructions. Genotyping of the discovery cohort was carried out by Sequenom's iPLEX SNP genotyping protocol using matrix-assisted laser desorption/ionization time of flight mass spectrometry on the MassArray Analyzer 4 system (Sequenom Inc., San Diego, CA, USA). SNP genotyping of the replication cohort was performed using a SNPscan Kit (Cat\#:G0104, Genesky Biotechnologies Inc., Shanghai, China) as described previously ${ }^{25}$. The SNPscan genotyping technology is based on double ligation and multiplex fluorescence polymerase chain reactions. As a quality control measure, we genotyped $5 \%$ of the samples in duplicate to check for concordance using the same method.

Statistical analyses. Comparisons of the demographic characteristics between cases and controls were conducted using Pearson's chi-squared test for the dichotomous variables and the $t$ test for the continuous variables. Hardy-Weinberg equilibrium (HWE) was assessed using the Pearson's chi-squared test. Unconditional logistic regression analyses were performed to test the association of each SNP with TB case/control status, adjusting for age and gender, under different genetic models (codominant, dominant, recessive and overdominant genetic models). All analyses were performed using the Statistical Package for the Social Sciences release 19.0 (SPSS Inc., Chicago, IL, USA). The association between haplotypes and TB susceptibility were also analyzed using unconditional logistic regression analyses, adjusting for age and gender, using SNPstats (http://bioinfo.iconcologia.net/ snpstats/start.htm).

The meta-analyses were performed with STATA version 12.0 (StataCorp, College Station, Texas). Following the procedure reported by Thakkinstian et al. ${ }^{26}$, pairwise differences were used to determine the most appropriate 


\begin{tabular}{|c|c|c|c|c|c|c|}
\hline SNP & Genetic model & Genotype & Controls, n (\%) & TB patients, n (\%) & OR $(95 \% \mathrm{CI})^{\mathrm{a}}$ & $P^{\text {a }}$ value \\
\hline \multirow{9}{*}{ rs9932581 } & \multirow{3}{*}{ Codominant } & TT & $209(34.6 \%)$ & $224(35.4 \%)$ & 1.00 & - \\
\hline & & TC & $289(47.9 \%)$ & $295(46.7 \%)$ & $1.00(0.99-1.01)$ & 0.37 \\
\hline & & CC & $106(17.6 \%)$ & $113(17.9 \%)$ & $1.17(0.86-1.60)$ & 0.31 \\
\hline & \multirow{2}{*}{ Dominant } & TT & $209(34.6 \%)$ & $224(35.4 \%)$ & 1.00 & - \\
\hline & & $\mathrm{CC}+\mathrm{TC}$ & $395(65.4 \%)$ & $408(64.6 \%)$ & $0.97(0.76-1.22)$ & 0.77 \\
\hline & \multirow{2}{*}{ Recessive } & $\mathrm{TC}+\mathrm{TT}$ & $498(82.5 \%)$ & $519(82.1 \%)$ & 1.00 & - \\
\hline & & $\mathrm{CC}$ & $106(17.6 \%)$ & $113(17.9 \%)$ & $1.02(0.76-1.37)$ & 0.88 \\
\hline & \multirow{2}{*}{ Overdominant } & $\mathrm{TT}+\mathrm{CC}$ & $315(52.1 \%)$ & $337(53.3 \%)$ & 1.00 & - \\
\hline & & TC & $289(47.9 \%)$ & $295(46.7 \%)$ & $0.96(0.76-1.20)$ & 0.69 \\
\hline \multirow{9}{*}{ rs3794624 } & \multirow{3}{*}{ Codominant } & GG & $419(70.5 \%)$ & $471(75.0 \%)$ & 1.00 & - \\
\hline & & GA & $167(28.1 \%)$ & $140(22.3 \%)$ & $0.74(0.57-0.96)$ & 0.03 \\
\hline & & $\mathrm{AA}$ & $8(1.4 \%)$ & $17(2.7 \%)$ & $1.38(0.90-2.11)$ & 0.14 \\
\hline & \multirow{2}{*}{ Dominant } & GG & $419(70.5 \%)$ & $471(75.0 \%)$ & 1.00 & - \\
\hline & & $\mathrm{AA}+\mathrm{GA}$ & $175(29.5 \%)$ & $157(25.0 \%)$ & $0.80(0.62-1.02)$ & 0.08 \\
\hline & \multirow{2}{*}{ Recessive } & $\mathrm{GA}+\mathrm{GG}$ & $586(98.7 \%)$ & $611(97.3 \%)$ & 1.00 & - \\
\hline & & $\mathrm{AA}$ & $8(1.4 \%)$ & $17(2.7 \%)$ & $2.05(0.88-4.80)$ & 0.09 \\
\hline & \multirow{2}{*}{ Overdominant } & $\mathrm{GG}+\mathrm{AA}$ & $427(71.9 \%)$ & $488(77.7 \%)$ & 1.00 & - \\
\hline & & GA & $167(28.1 \%)$ & $140(22.3 \%)$ & $0.73(0.56-0.95)$ & 0.02 \\
\hline \multirow{9}{*}{ rs 4673} & \multirow{3}{*}{ Codominant } & GG & $506(83.6 \%)$ & $549(86.9 \%)$ & 1.00 & - \\
\hline & & GA & $97(16.0 \%)$ & $82(13.0 \%)$ & $0.78(0.57-1.07)$ & 0.12 \\
\hline & & AA & $2(0.3 \%)$ & $1(0.2 \%)$ & $0.67(0.20-2.23)$ & 0.51 \\
\hline & \multirow{2}{*}{ Dominant } & GG & $506(83.6 \%)$ & $549(86.9 \%)$ & 1.00 & - \\
\hline & & $\mathrm{GA}+\mathrm{AA}$ & $99(16.4 \%)$ & $83(13.1 \%)$ & $0.77(0.56-1.06)$ & 0.11 \\
\hline & \multirow{2}{*}{ Recessive } & $\mathrm{GG}+\mathrm{GA}$ & $603(99.7 \%)$ & $631(99.8 \%)$ & 1.00 & - \\
\hline & & AA & $2(0.3 \%)$ & $1(0.2 \%)$ & $0.47(0.04-5.20)$ & 0.53 \\
\hline & \multirow{2}{*}{ Overdominant } & $\mathrm{GG}+\mathrm{AA}$ & $508(84.0 \%)$ & $550(87.0 \%)$ & 1.00 & - \\
\hline & & GA & $97(16.0 \%)$ & $82(13.0 \%)$ & $0.78(0.57-1.07)$ & 0.13 \\
\hline
\end{tabular}

Table 1. Association between CYBA SNPs and TB susceptibility in the discovery cohort. Abbreviation: SNP,

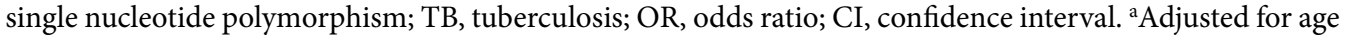
and gender in a logistic regression model.

genetic model for the meta-analysis. The procedure is as follows (assuming "A" as the risk allele compared with "a" allele; odds ratio $(\mathrm{OR})_{1}, \mathrm{OR}_{2}$ and $\mathrm{OR}_{3}$ representing comparisons of $\mathrm{AA}$ vs. aa, Aa vs. aa and $\mathrm{AA}$ vs. Aa): (a) $\mathrm{OR}_{1}=\mathrm{OR}_{3} \neq 1$ and $\mathrm{OR}_{2}=1$, a recessive model is suggested; (b) $\mathrm{OR}_{1}=\mathrm{OR}_{2} \neq 1$ and $\mathrm{OR}_{3}=1$, a dominant model is suggested. (c) $\mathrm{OR}_{2}=1 / \mathrm{OR}_{3} \neq 1$ and $\mathrm{OR}_{1}=1$, an overdominant model is suggested. (d) $\mathrm{OR}_{1}<\mathrm{OR}_{2}<1$ and $\mathrm{OR}_{1}<\mathrm{OR}_{3}<1$ (or $\mathrm{OR}_{1}>\mathrm{OR}_{2}>1$ and $\mathrm{OR}_{1}>\mathrm{OR}_{3}>1$ ), a codominant model is suggested.

$\chi^{2}$ based Q statistics and the $I^{2}$ test were used to assess the between-study heterogeneity. These tests indicated a lack of heterogeneity between the discovery and replication sets, and therefore a fixed effects model (Mantel-Haenszel's method) was used in the meta-analysis ${ }^{27}$.

Adjusted OR estimates and 95\% CIs on a natural logarithmic scale were used to assess the strength of association between SNP and TB susceptibility. A $p$ value $<0.05$ was considered statistically significant in all the above statistical analyses except the between-study heterogeneity analysis.

\section{Results}

Discovery cohort. The discovery cohort was composed of $636 \mathrm{~TB}$ cases ( 324 males and 312 females, mean age $=36.77 \pm 15.71)$ and 608 controls ( 302 males and 306 females, mean age $=37.14 \pm 15.68)$ from the Chinese Han population. There was no significant difference in either age or gender between two groups, as the cases and controls were matched on these variables. No deviations from HWE were detected in the control subjects $(p>0.05)$. The genotype call rates were $99.4 \%$ for rs $9932581,98.2 \%$ for $r s 3794624$ and $99.4 \%$ for rs 4673 .

The main results of the association between SNPs in CYBA and TB risk are presented in Table 1. After adjusting for age and gender, we found the A allele of the rs3794624 polymorphism to be a significant protective factor against TB in the Chinese Han population under two genetic models (GA vs. GG: OR 0.74, 95\% CI 0.57-0.96, $p=0.03$; GA vs. GG+AA: OR $0.73,95 \%$ CI $0.56-0.95, p=0.02$ ). There was no significant association identified between rs9932581 T $>$ C or rs4673 T $>$ C polymorphisms and TB susceptibility under any genetic model.

Five CYBA haplotypes (GTG, GCG, ACG, ATG and GTA) were identified, with frequencies more than 0.03 (Table 2). We observed no significant differences of $C Y B A$ haplotype frequencies between TB and control groups, when adjusting for age and gender.

Replication cohort. To validate the association of rs3794624 with TB risk we genotyped an independent cohort of Chinese Tibetans, including $613 \mathrm{~TB}$ cases $(327$ males and 286 females, mean age $=34.54 \pm 13.87)$ and 603 controls ( 333 males and 270 females, mean age $=34.62 \pm 13.84$ ), with no difference in age and gender ratio 


\begin{tabular}{|l|c|c|c|c|c|}
\hline rs3794624 & rs9932581 & rs4673 & Frequency & OR $(\mathbf{9 5 \%} \text { CI })^{\mathbf{a}}$ & P $^{\text {a }}$ value \\
\hline G & T & G & 0.479 & 1.00 & - \\
\hline G & C & G & 0.303 & $0.95(0.77-1.16)$ & 0.59 \\
\hline A & C & G & 0.081 & $1.02(0.73-1.43)$ & 0.90 \\
\hline A & T & G & 0.062 & $0.71(0.48-1.07)$ & 0.10 \\
\hline G & T & A & 0.045 & $0.81(0.51-1.30)$ & 0.39 \\
\hline
\end{tabular}

Table 2. Haplotype analysis of CYBA SNPs in association with the risk of TB. Abbreviation: OR, odds

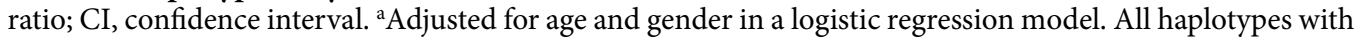
frequencies $<0.03$ were ignored in the analysis.

\begin{tabular}{|c|c|c|c|c|c|c|}
\hline SNP & Genetic model & Genotype & Controls, $\mathbf{n}(\%)$ & TB patients, n (\%) & OR $(95 \% \mathrm{CI})^{\mathrm{a}}$ & $P^{a}$ value \\
\hline \multirow{9}{*}{ rs3794624 } & \multirow{3}{*}{ Codominant } & GG & $472(78.4 \%)$ & $514(83.8 \%)$ & 1.00 & - \\
\hline & & GA & $124(20.6 \%)$ & $92(15.0 \%)$ & $0.68(0.51-0.92)$ & 0.01 \\
\hline & & $\mathrm{AA}$ & $6(1.0 \%)$ & $7(1.1 \%)$ & $1.00(0.99-1.01)$ & 0.92 \\
\hline & \multirow{2}{*}{ Dominant } & GG & $472(78.4 \%)$ & $514(83.8 \%)$ & 1.00 & - \\
\hline & & $\mathrm{AA}+\mathrm{GA}$ & $130(21.6 \%)$ & $99(16.1 \%)$ & $0.70(0.52-0.93)$ & 0.02 \\
\hline & \multirow{2}{*}{ Recessive } & $\mathrm{GA}+\mathrm{GG}$ & $596(99 \%)$ & $606(98.9 \%)$ & 1.00 & - \\
\hline & & $\mathrm{AA}$ & $6(1.0 \%)$ & $7(1.1 \%)$ & $1.12(0.37-3.37)$ & 0.83 \\
\hline & \multirow{2}{*}{ Overdominant } & $\mathrm{GG}+\mathrm{AA}$ & $478(79.4 \%)$ & $521(85.0 \%)$ & 1.00 & - \\
\hline & & GA & $124(20.6 \%)$ & $92(15.0 \%)$ & $0.68(0.51-0.92)$ & 0.01 \\
\hline
\end{tabular}

Table 3. Association between $C Y B A$ rs3794624 and TB susceptibility in the replication cohort.

Abbreviations: SNP, single nucleotide polymorphism; TB, tuberculosis; OR, odds ratio; CI, confidence interval.

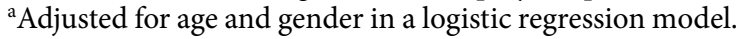

\begin{tabular}{|c|c|c|c|}
\hline Study & OR1 $(95 \% \text { CI })^{\mathrm{a}}$ & OR2 $(95 \% \text { CI })^{a}$ & OR3 $(95 \% \text { CI })^{\mathrm{a}}$ \\
\hline Discovery cohort & $1.38(0.90-2.11)$ & $0.74(0.57-0.96)$ & $2.55(1.07-6.90)$ \\
\hline Replication cohort & $1.03(0.59-1.78)$ & $0.68(0.51-0.92)$ & $1.57(0.50,4.88)$ \\
\hline Overall & $1.24(0.88-1.73)$ & $0.71(0.59-0.87)$ & $2.10(1.02-4.32)$ \\
\hline
\end{tabular}

Table 4. Pairwise comparisons of rs3794624 genotypes and TB susceptibility before determining the best genetic model. OR, odds ratio; CI, confidence interval. aAdjusted for age and gender in a logistic regression model.

between the groups. No deviation from HWE was detected in the control subjects $(p>0.05)$. The genotype call rate for rs 3794624 was $99.9 \%$.

The replication cohort showed strong supporting evidence for the association between rs3794624 polymorphism and TB risk. After adjusting for age and gender, we found the A allele of the rs3794624 polymorphism to be a significant protective factor against TB in the Chinese Tibetan population under three genetic models: overdominant (GA vs. GG+AA: OR 0.68, 95\% CI 0.51-0.92, $\mathrm{p}=0.01$ ), heterozygous (GA vs. GG: OR 0.68, 95\% CI $0.51-0.92, p=0.01$ ) and dominant (AA+GA vs. GG: OR 0.70, 95\% CI 0.52-0.93, $p=0.02$ ), as shown in Table 3.

Meta analysis of the two included cohorts. We included both discovery and replication cohorts in the following meta-analysis. The pooled $\mathrm{OR}_{1}, \mathrm{OR}_{2}$ and $\mathrm{OR}_{3}$ of $\mathrm{rs} 3794624$ genotypes with $\mathrm{TB}$ susceptibility are shown in Table 4. The Wald test showed that $\mathrm{OR}_{2}$ and $\mathrm{OR}_{3}$ were both significant ( $p=0.001$ and 0.04 , respectively), while $\mathrm{OR}_{1}$ was not significant $(p=0.22)$. As a result, the overdominant model was then determined to be the most appropriate genetic model. The pooling analysis provided robust evidence for the association of rs $3794624 \mathrm{GA}$ heterozygote with decreased TB risk without any evidence of heterogeneity (GA vs. GG+AA: OR 0.71, 95\% CI $0.58-0.86, p=0.001$ ), as shown in Fig. 1 .

\section{Discussion}

The NADPH oxidase complex is a key enzyme that catalyzes the production of ROS, which mediate oxygen-dependent killing of microorganisms, such as MTB, and also play a role in the injurious effects of oxidative stress on body tissues or organs ${ }^{28-30}$. Numerous reports demonstrating a high prevalence of BCG complications and TB risk in chronic granulomatous disease provide a strong rationale for the critical role of NADPH oxidase in controlling TB in humans ${ }^{9-13}$. The function of the NADPH oxidase complex is largely dependent upon its regulatory subunit p22phox (encoded by $C Y B A$ ) which increase the stability the large subunit and undertakes the role as docking unit for the cytosolic factors ${ }^{15}$. Up until now, no other studies have address the role of polymorphisms in CYBA and TB susceptibility. 


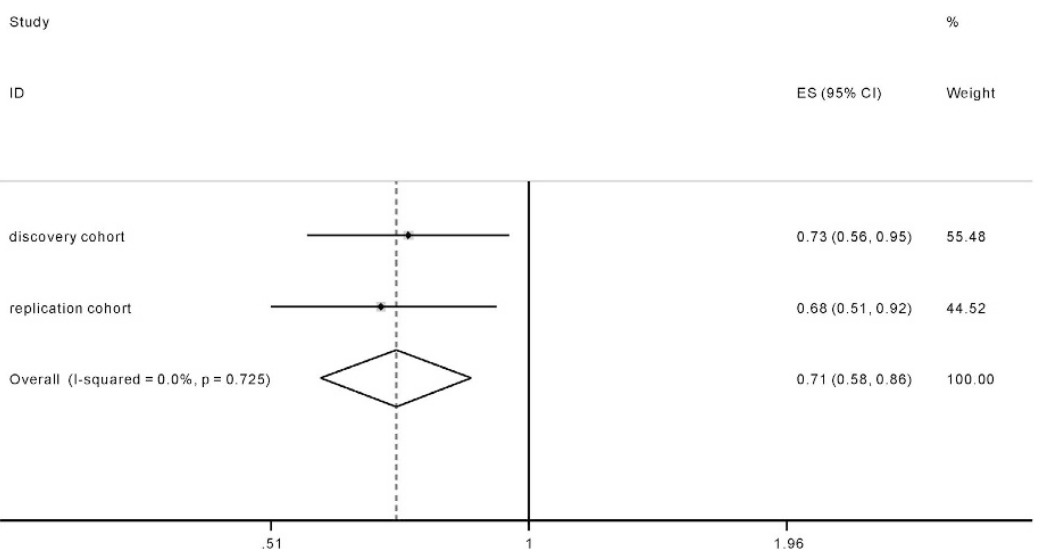

Figure 1. Forrest plot of the association between rs3794624 and TB risk under the overdominant model.

\section{Data supporting chr16:88717073 (rs3794624)}

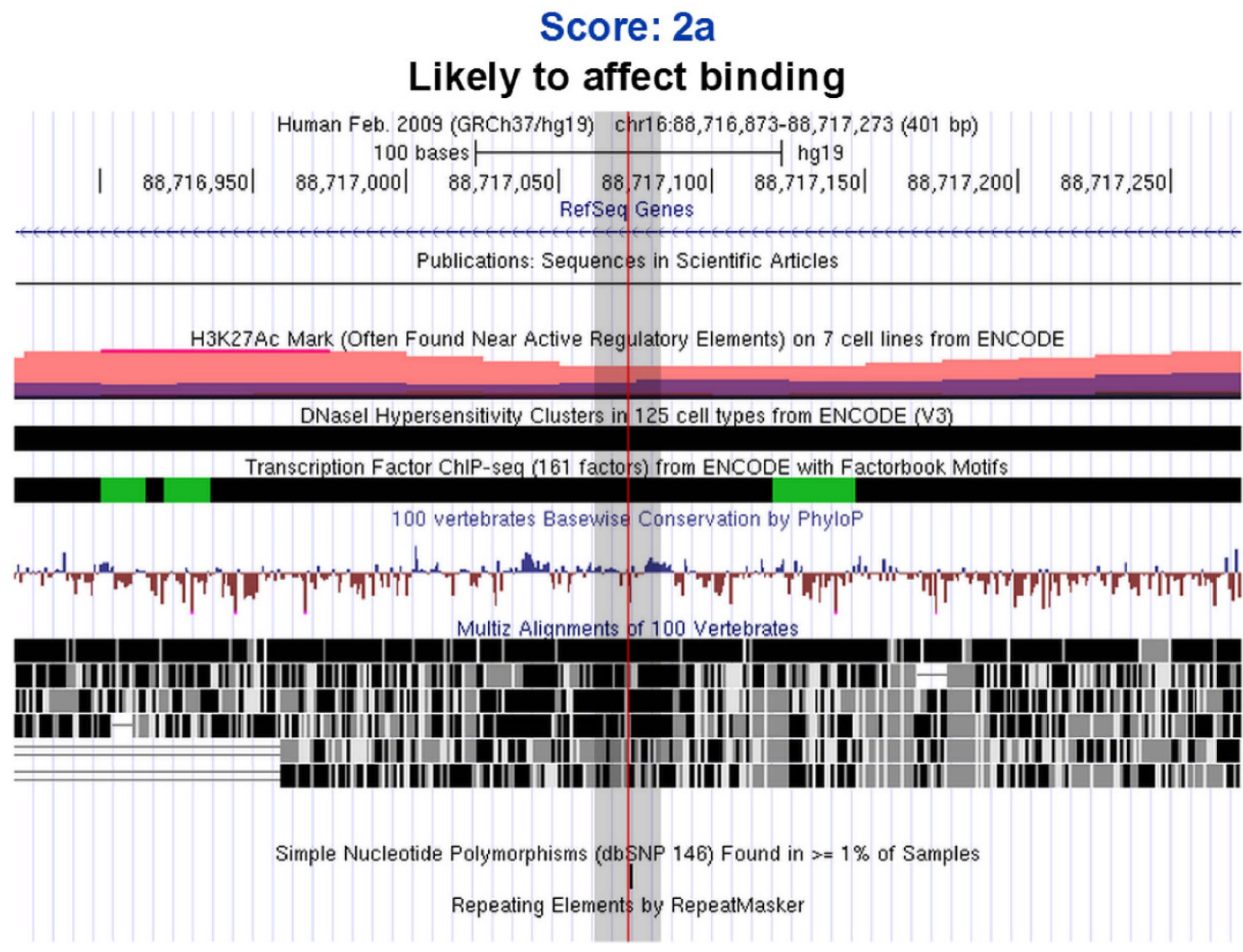

Figure 2. Functional prediction result of rs3794624 by Regulome DB.

$C Y B A$ is located on the long arm of chromosome $16(16 \mathrm{q} 24)$ and spans approximately $8.5 \mathrm{~kb}$, including six exons and five introns. Three SNPs (rs9932581 T > C, rs3794624 G > A and rs4673 T > C) were included in our initial association analysis. rs 9932581 and $\mathrm{rs} 4673$ are both potential functional SNPs, based on in silico functional prediction and literature review ${ }^{16,20}$, but neither showed significant association with susceptibility to TB in our Chinese Han population. However, our study found an association between rs 3794624 in CYBA with decreased TB susceptibility in two independent Chinese cohorts and in the meta-analysis.

rs 3794624 has been associated with clinical outcomes such as peripheral arterial disease ${ }^{19}$ and breast cancer risk $^{22}$ suggesting a possible functional role. In addition, both of the above-mentioned diseases are considered to be ROS-related. However, we did not find any study of this polymorphism that directly demonstrated a functional effect. rs3794624 is located in the first intron of $C Y B A$ and therefore may lead to the alternative splicing of the transcript or expression level change. The in silico functional prediction for rs3794624 suggests a potential 
functional significance of this polymorphism site. As shown in Fig. 2, rs3794624 G > A was predicted to be functional SNP in the Regulome DB (LSJU, Stanford, CA, USA) with a score of $2 \mathrm{a}$, which is annotated as to be likely to affect binding with transcription factors and the supporting data of this locus were as follows: TF binding+ matched TF motif + matched DNase Footprint+DNase peak. Thus, further investigation of the functional significance of rs3794624 is needed.

The association of rs3794624 with susceptibility to TB may also be due to LD with another polymorphism that has a functional effect. rs3794624 is in $\mathrm{LD}\left(\mathrm{r}^{2} \geq 0.7\right)$ with three other CYBA polymorphisms (rs33997949, rs13306296 and rs35601559) in the East Asian populations of the 1000 Genomes Project (http://www.1000genomes.org/). However, none of these three SNPs have any obvious functional significance.

As haplotypes are often more relevant than individual $\mathrm{SNPs}^{31}$, we further did haplotype analysis of CYBA SNPs in association with the risk of TB. However no statistically significant association was identified. This result may be ascribed to the fact that the protective effect of $C Y B A$ polymorphisms manifested as a heterozygote advantage over individuals carrying either homozygous genotype rather than the impact of a haplotype, which is the combination of alleles in different sites. We speculate that heterozygotes for rs3794624 are more fit in terms of natural selection because of their intermediate phenotype with respect to enzyme activity. As mentioned above, ROS released by NADPH oxidase complex is a double-edged sword in MTB immunity, as they can lead to the elimination of this pathogen but also result in injury due to oxidative stress. Therefore, the GA genotype of rs3794624 may lead to neither too much nor too little ROS production and provide the optimal protective effect in TB. In this regard, it is worth mentioning that Tarazona-Santos et al. conducted a resequencing analysis of CYBA in 102 individuals with different ethnicities, and reached similar conclusions to ours ${ }^{32}$. In their report, they found $C Y B A$ was characterized by high diversity and high frequency of common polymorphisms in Europeans, which was considered to be the result of balancing natural selection. Heterozygote advantage was speculated to be the biological mechanism underlying balancing natural selection. That is to say, heterozygous individuals producing an inter-mediate level of ROS, had a survival advantage during human evolution. Combining our research, we can infer that MTB-driven selective pressure may be one of the factors that promoted the balancing selection of CYBA.

Our research has several strengths. Firstly, to our knowledge, this is the first case-control study to investigate CYBA polymorphisms and TB susceptibility. Our study has shown rs3794624 as protective factor against TB in the Chinese population. Secondly, our study included two large cohorts (Chinese Han and Chinese Tibetan). The consistent results from two independent populations with different ethnic backgrounds considerably increase confidence that the association is not due to type I error.

Nevertheless, some limitations in this research should also be addressed. First, we did not perform functional validation of the associated SNP. As a result, the mechanism underlying the genetic association result is still unknown. Second, we did not correct our results for multiple testing, which may increase the chance of type I errors. However, the results from the replication cohort and the pooled analysis would survive such correction.

\section{Conclusions}

In conclusion, we have demonstrated an association between the GA genotype of rs3794624 in CYBA with decreased TB susceptibility, which suggests new avenues for exploring the role of oxygen-dependent innate immunity against MTB in the development of TB. However, further analyses are needed to fully validate these findings in other ethnic populations and to reveal the potential function of this SNP.

\section{References}

1. World Health Organization. Global tuberculosis report 2015 Available from: http://www.who.int/tb/publications/global_report/en/ Accessed on 30th, May (2015).

2. Rosman, M. D. \& Oner-Eyupoglu, A. F. Clinical Presentation and Treatment of Tuberculosis In Fishman's Pulmonary Diseases and Disorders (ed. Fishman, A. P.) 2483-2502 (McGraw-Hill, 1998).

3. Bellamy, R. Susceptibility to mycobacterial infections: the importance of host genetics. Genes and immunity 4, 4-11, doi: 10.1038/ sj.gene.6363915 (2003).

4. Caws, M. et al. The influence of host and bacterial genotype on the development of disseminated disease with Mycobacterium tuberculosis. PLoS pathogens 4, e1000034, doi: 10.1371/journal.ppat.1000034 (2008).

5. Slama, K. et al. Tobacco and tuberculosis: a qualitative systematic review and meta-analysis. The international journal of tuberculosis and lung disease: the official journal of the International Union against Tuberculosis and Lung Disease 11, 1049-1061 (2007).

6. Korbel, D. S., Schneider, B. E. \& Schaible, U. E. Innate immunity in tuberculosis: myths and truth. Microbes and infection/Institut Pasteur 10, 995-1004, doi: 10.1016/j.micinf.2008.07.039 (2008).

7. Meyer, C. G. \& Thye, T. Host genetic studies in adult pulmonary tuberculosis. Seminars in immunology 26, 445-453, doi: 10.1016/j. smim.2014.09.005 (2014)

8. Deffert, C., Cachat, J. \& Krause, K. H. Phagocyte NADPH oxidase, chronic granulomatous disease and mycobacterial infections. Cellular microbiology 16, 1168-1178, doi: 10.1111/cmi.12322 (2014).

9. Mouy, R., Fischer, A., Vilmer, E., Seger, R. \& Griscelli, C. Incidence, severity, and prevention of infections in chronic granulomatous disease. The Journal of pediatrics 114, 555-560 (1989).

10. Movahedi, M. et al. Chronic granulomatous disease: a clinical survey of 41 patients from the Iranian primary immunodeficiency registry. International archives of allergy and immunology 134, 253-259, doi: 10.1159/000078774 (2004).

11. von Goessel, H., Hossle, J. P., Seger, R. \& Gungor, T. Characterization of 17 new cases of X-linked chronic granulomatous disease with seven novel mutations in the CYBB gene. Experimental hematology 34, 528-535, doi: 10.1016/j.exphem.2006.01.005 (2006).

12. Bustamante, J. et al. BCG-osis and tuberculosis in a child with chronic granulomatous disease. The Journal of allergy and clinical immunology 120, 32-38, doi: 10.1016/j.jaci.2007.04.034 (2007).

13. Stasia, M. J. et al. Characterization of six novel mutations in the CYBB gene leading to different sub-types of X-linked chronic granulomatous disease. Human genetics 116, 72-82, doi: 10.1007/s00439-004-1208-5 (2005).

14. Lassegue, B., San Martin, A. \& Griendling, K. K. Biochemistry, physiology, and pathophysiology of NADPH oxidases in the cardiovascular system. Circulation research 110, 1364-1390, doi: 10.1161/CIRCRESAHA.111.243972 (2012). 
15. Ushio-Fukai, M., Zafari, A. M., Fukui, T., Ishizaka, N. \& Griendling, K. K. p22phox is a critical component of the superoxidegenerating NADH/NADPH oxidase system and regulates angiotensin II-induced hypertrophy in vascular smooth muscle cells. The Journal of biological chemistry 271, 23317-23321 (1996).

16. Moreno, M. U. et al. Preliminary characterisation of the promoter of the human p22(phox) gene: identification of a new polymorphism associated with hypertension. FEBS letters 542, 27-31 (2003).

17. Yamada, Y. et al. Prediction of the risk of myocardial infarction from polymorphisms in candidate genes. The New England journal of medicine 347, 1916-1923, doi: 10.1056/NEJMoa021445 (2002).

18. Vibhuti, A. et al. CYP1A1, CYP1A2 and CYBA gene polymorphisms associated with oxidative stress in COPD. Clinica chimica acta; international journal of clinical chemistry 411, 474-480, doi: 10.1016/j.cca.2009.12.018 (2010).

19. Murabito, J. M. et al. Association between chromosome $9 \mathrm{p} 21$ variants and the ankle-brachial index identified by a meta-analysis of 21 genome-wide association studies. Circulation. Cardiovascular genetics 5, 100-112, doi: 10.1161/CIRCGENETICS.111.961292 (2012).

20. Petrovic, D. Association of the $-262 \mathrm{C} / \mathrm{T}$ polymorphism in the catalase gene promoter and the C242T polymorphism of the NADPH oxidase P22phox gene with essential arterial hypertension in patients with diabetes mellitus type 2. Clinical and experimental hypertension 36, 36-39, doi: 10.3109/10641963.2013.783051 (2014).

21. Niemiec, P. et al. The $-930 \mathrm{~A}>\mathrm{G}$ polymorphism of the $C Y B A$ gene is associated with premature coronary artery disease. A casecontrol study and gene-risk factors interactions. Molecular biology reports 41, 3287-3294, doi: 10.1007/s11033-014-3191-9 (2014).

22. Seibold, P. et al. Polymorphisms in oxidative stress-related genes and postmenopausal breast cancer risk. International journal of cancer 129, 1467-1476, doi: 10.1002/ijc.25761 (2011).

23. Sun, Q., Yin, Y., Zhu, Z. \& Yan, Z. Association of the C242T polymorphism in the NAD(P)H oxidase P22 phox gene with type 2 diabetes mellitus risk: a meta-analysis. Current medical research and opinion 30, 415-422, doi: 10.1185/03007995.2013.858620 (2014).

24. Fang, S., Wang, L. \& Jia, C. Association of p22phox gene C242T polymorphism with coronary artery disease: a meta-analysis. Thrombosis research 125, e197-201, doi: 10.1016/j.thromres.2010.01.001 (2010).

25. Du, W. et al. A rapid method for simultaneous multi-gene mutation screening in children with nonsyndromic hearing loss. Genomics 104, 264-270, doi: 10.1016/j.ygeno.2014.07.009 (2014).

26. Thakkinstian, A., McElduff, P., D'Este, C., Duffy, D. \& Attia, J. A method for meta-analysis of molecular association studies. Statistics in medicine 24, 1291-1306, doi: 10.1002/sim.2010 (2005).

27. Mantel, N. \& Haenszel, W. Statistical aspects of the analysis of data from retrospective studies of disease. Journal of the National Cancer Institute 22, 719-748 (1959).

28. Billings, F. T. t., Ball, S. K., Roberts, L. J. 2nd \& Pretorius, M. Postoperative acute kidney injury is associated with hemoglobinemia and an enhanced oxidative stress response. Free radical biology \& medicine 50, 1480-1487, doi: 10.1016/j.freeradbiomed.2011.02.011 (2011).

29. Hoidal, J. R. et al. Lung injury and oxidoreductases. Environmental health perspectives 106 Suppl 5, 1235-1239 (1998).

30. Griffith, B. et al. NOX enzymes and pulmonary disease. Antioxidants \& redox signaling 11, 2505-2516, doi: 10.1089/ARS.2009.2599 (2009).

31. Bedard, K. et al. Three common polymorphisms in the CYBA gene form a haplotype associated with decreased ROS generation. Human mutation 30, 1123-1133, doi: 10.1002/humu.21029 (2009).

32. Tarazona-Santos, E. et al. Evolutionary dynamics of the human NADPH oxidase genes CYBB, CYBA, NCF2, and NCF4: functional implications. Molecular biology and evolution 30, 2157-2167, doi: 10.1093/molbev/mst119 (2013).

\section{Acknowledgements}

This work was supported by the Research Fund from the National Scientific and Technological Major Project of China (Beijing, China) Grant 2012ZX10004-901; the National Natural Science Foundation of China (Beijing, China) Grants 81072432, 81170042, and 81370121; Science \& Technology Department of Sichuan Province (Chengdu, Sichuan, China) Grant 2012SZ0126.

\section{Author Contributions}

Conceived and designed the study: Q.Q.L., J.Q.H.; Analyzed the data: J.L., M.X., J.C.W., Y.W., S.Q.W., G.C., C.M.T., Y.T., Y.L.F., J.Q.H.; Wrote the manuscript: Q.Q.L., A.J.S., J.Q.H.

\section{Additional Information}

Competing financial interests: The authors declare no competing financial interests.

How to cite this article: Liu, Q. et al. Heterozygote Advantage of the rs3794624 Polymorphism in CYBA for Resistance to Tuberculosis in Two Chinese Populations. Sci. Rep. 6, 38213; doi: 10.1038/srep38213 (2016).

Publisher's note: Springer Nature remains neutral with regard to jurisdictional claims in published maps and institutional affiliations.

(c) (i) This work is licensed under a Creative Commons Attribution 4.0 International License. The images

or other third party material in this article are included in the article's Creative Commons license, unless indicated otherwise in the credit line; if the material is not included under the Creative Commons license, users will need to obtain permission from the license holder to reproduce the material. To view a copy of this license, visit http://creativecommons.org/licenses/by/4.0/

(C) The Author(s) 2016 